\title{
Caffeine is a potent stimulator of autophagy to reduce hepatic lipid content-a coffee for NAFLD?
}

C

offee drinking is a global phenomenon, with an estimated $>2.25$ billion cups of coffee consumed in the world every day. A myriad of health benefits across a wide range of diseases have been attributed to coffee consumption and much research has focussed on the effects of caffeine on the liver. Now, a new study published in Hepatology has investigated the mechanism of action of caffeine on lipid metabolism, finding that caffeine reduces lipid content in the liver via the autophagy-lysosomal pathway.

"Coffee is probably the world's most consumed drug and its consumption has been shown to be associated with reduced incidence of several liver diseases, including NAFLD," notes author Paul Yen, Duke-National University of Singapore Graduate Medical School, Singapore. "We and others have previously shown that autophagy has an important role in lipid metabolism and clearance in the mammalian liver, and therefore examined whether caffeine modulation of autophagy and lipid metabolism might explain the beneficial effects of caffeine in the liver," he continues.

The researchers used genetic, pharmacological and metabolomic approaches both in vitro and in vivo to study the effects of caffeine on the liver.

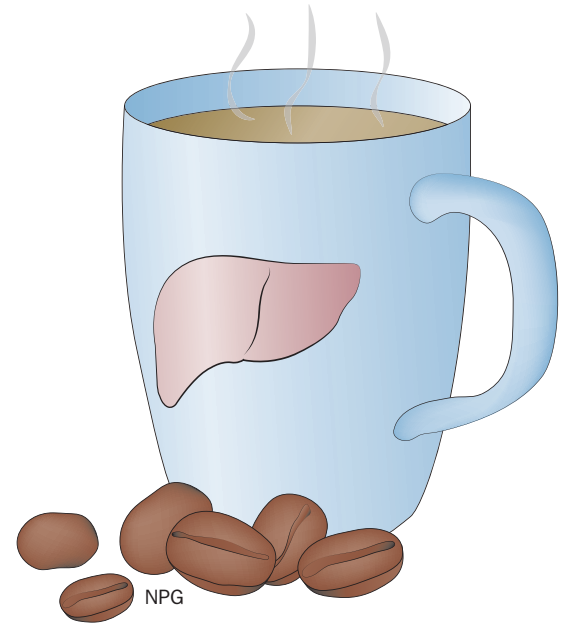

First, the researchers demonstrated that the autophagy-lysosomal pathway was involved in the reduction of intracellular lipids by caffeine in vitro; caffeine induced autophagosome formation in HepG2 cells at the same time as lipid clearance, and electron microscopy revealed co-localization of autophagy proteins and intracellular lipids in caffeine-treated cells. Knockdown of ATG5 (an important protein in autophagosome formation) using small interfering RNA led to a marked decrease in caffeine-mediated intracellular lipid reduction in vitro in hepatic cells.

Next, Yen, Sinha and colleagues investigated the metabolic effects of caffeine in animals. Caffeine treatment ( $30 \mathrm{mg} / \mathrm{kg}$ for $24 \mathrm{~h}$ in mice) increased fatty acid oxidation (as measured by respiratory exchange ratio). Moreover, metabolomic analysis confirmed increased flux through the beta-oxidation pathway and increased lipolysis upon caffeine treatment $(30 \mathrm{mg} / \mathrm{kg}$ for 3 days) in mice. By treating mice with chloroquine (a known autophagy inhibitor) concomitantly with caffeine, the investigators confirmed that autophagy is essential for caffeine-stimulated beta-oxidation.

Finally, the effects of caffeine on autophagy and hepatosteatosis were investigated in an animal model of dietinduced obesity. Mice were fed either a normal or high-fat diet for 4 weeks; the diet was then continued in the absence or presence of caffeine for a further 4 weeks. Interestingly, caffeine-treated mice on the high-fat diet had substantially lower percentage fat mass and higher percentage lean mass than mice fed the high-fat diet alone, indicating that caffeine inhibited the weight gain induced by a high-fat diet.

In addition, caffeine-treated mice had little or no intrahepatic lipid accumulation even whilst on a high-fat diet; hepatic lipid content was substantially increased in mice fed the high-fat diet alone compared with mice on the normal diet.
Crucially, electron microscopy images revealed almost no cytosolic lipid present in liver sections from caffeine-treated mice fed a high-fat diet, with lipids almost exclusively observed within the autolysosomal compartment and indeed often surrounded by double membranes, indicative of engulfed lipid-laden autophagosomes to be cleared by the cell.

"Our findings support a newly recognized role of autophagy in lipid metabolism and might help explain the observed association of caffeine and NAFLD in humans," says Yen, who hopes that further research will lead to the development of "caffeine-like drugs" for liver disease and that the "full benefits of caffeine and related therapeutics in humans" will be revealed.

Whether we will see clinicians prescribing a 'cup of joe' to treat NAFLD remains to be seen, but clearly the potent effects of caffeine in the liver warrant further investigation to open up new avenues of treatment for liver disease. In fact, a recent systematic review published in Liver International investigated the influence of coffee on a wide range of liver diseases, and the findings provide more support for the therapeutic use of coffee. Coffee consumption was associated with a plethora of benefits in chronic liver disease-decreased risk of progression to cirrhosis, reduced rate of development of hepatocellular carcinoma and an inverse association with severity of steatohepatitis, among others-with the study authors concluding that "daily consumption should be encouraged" in patients with chronic liver diseases.

\section{Katrina Ray}

Original article Sinha, R. A. Caffeine stimulates hepatic lipid metabolism via autophagy-lysosomal pathway. Hepatology doi:10.1002/hep.26667

Further reading Saab, S. et al. Impact of coffee on liver diseases: a systematic review. Liver Int. doi:10.1111/ liv. 12304 